\section{Asymmetric reduction of ketones with recombinant $E$. coli whole cells in neat substrates}

\author{
Andre Jakoblinnert, ${ }^{a}$ Radoslav Mladenov, ${ }^{a}$ Albert Paul, ${ }^{a}$ Fabrizio Sibilla, ${ }^{a}$ \\ Ulrich Schwaneberg, ${ }^{a}$ Marion B. Ansorge-Schumacher*b and Pablo Domínguez de María*c
}

Received 8th July 2011, Accepted 5th October 2011

DOI: $10.1039 / \mathrm{clcc14097c}$

The asymmetric reduction of ketones is performed by using lyophilized whole cells in neat substrates with defined water activity $\left(a_{w}\right)$. Ketones and alcohols prone to be unstable in aqueous media can now be converted via biocatalysis.

Biocatalysis is receiving increasing attention as a powerful synthetic tool providing environmentally-friendly reaction conditions with high regio- and stereoselectivities. ${ }^{1}$ However, a broader extension of industrial biocatalysis is still hampered due to several reasons, e.g. restricted enzyme availabilities, insufficient productivities and stabilities, time- and materialintensive work-ups in aqueous media-often leading to waste production - as well as costs related to catalysts and cofactors. ${ }^{2}$ Low product concentrations resulting from a limited water solubility of many organic compounds can be, to some extent, enhanced by reaction engineering using organic co-solvents or water-organic biphasic systems. ${ }^{3,4}$ On the other hand, process stability of biocatalysts can be increased and costs for catalysts and cofactors can be reduced by the use of whole cells rather than isolated enzymes. 5,6

For the industrial performance of the asymmetric reduction of prochiral ketones by alcohol dehydrogenases the demand for expensive nicotinamide cofactors plays a particular role in cell-free systems. ${ }^{7}$ Hence, the use of whole cells exhibits as a major advantage that cofactors can be intrinsically recycled via the coupling of a second substrate or by a second enzyme. ${ }^{8}$ Few reduction reactions have been reported which work in water-organic mixtures showing exceedingly high substrate loadings and no or only little demand for external cofactors. ${ }^{7,9,10}$ However, in such water-based systems emulsions may be formed, decreasing isolated yields and requiring tedious product separation. Moreover, the general applicability of the latter system is restricted when substrates and products with low stability in aqueous environments are involved.

\footnotetext{
${ }^{a}$ Lehrstuhl für Biotechnology, RWTH Aachen University,

Worringer Weg 1, D-52074 Aachen, Germany

${ }^{b}$ Institute of Chemistry, Department of Enzyme Technology (TC4),

Technische Universität Berlin, Straße des 17. Juni 124, D-10623

Berlin,Germany.E-mail: m.ansorge@chem.tu-berlin.de;

Fax: + 493031422261 ; Tel: + 493031422127

${ }^{c}$ Institut für Technische und Makromolekulare Chemie (ITMC),

RWTH Aachen University, Worringer Weg 1, D-52074 Aachen,

Germany.E-mail: Dominguez@itmc.rwth-aachen.de;

Fax: + $492418022177 ;$ Tel: + 492418020468
}

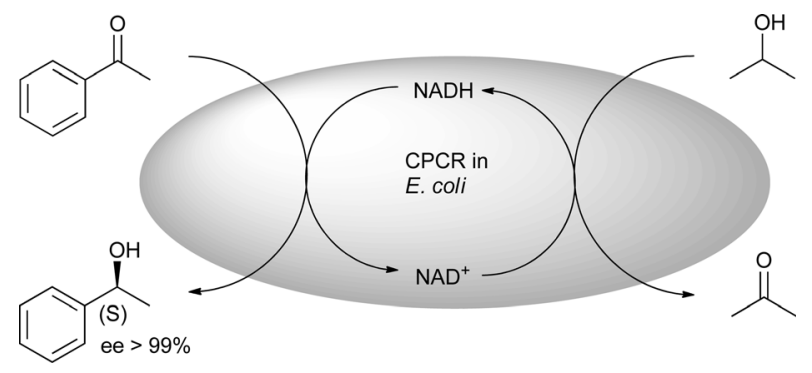

Fig. 1 Biocatalytic reduction of acetophenone using lyophilized E. coli cells with overexpressed carbonyl reductase in neat substrates with isopropanol-coupled cofactor regeneration.

Herein we show that $E$. coli whole cells, overexpressing a NADH-dependent carbonyl reductase, are able to perform enantioselective carbonyl reduction in quantitative yields in neat substrates without external cofactor addition (Fig. 1).

The reaction setup only requires a substrate, a co-substrate, and the biocatalyst at a defined water activity (see below). Despite its potentiality, there are only few examples using dried yeast cells in organic solvents, ${ }^{11-14}$ as well as lyophilized E. coli overexpressing an alcohol dehydrogenase from Rhodococcus ruber in $99 \%$ (v/v) isopropanol. ${ }^{15}$ In that latter example, however, cells displayed little catalytic activity, presumably due to cell rehydration before using them in the micro-aqueous system. $^{15}$

For a model reaction, acetophenone as a substrate and isopropanol as an ancillary co-substrate for cofactor regeneration were used (Fig. 1). The catalyst was a NADH-dependent carbonyl reductase from Candida parapsilosis $(\mathrm{CPCR})^{16-18}$ overexpressed and directly applied in E. coli whole cells. In this highly non-natural environment the biocatalyst was able to effectively produce $(S)$-phenylethanol (ee $>99 \%$; >98\% conversion) with high productivities (300-500 $\mathrm{g} \mathrm{L}^{-1}$ ) (Fig. 2). ${ }^{19}$ Compared to existing production processes, the developed reaction system has advantage in simplicity, economics (cheap starting material, no NADH addition, very limited waste production, high added value), scalability and high yields. ${ }^{7,9,10,15}$ Moreover, a key feature is the straightforward work-up as the whole cells can easily be separated and product downstream requires only evaporating the co-substrate and the co-product.

A further study of the whole-cell-catalyzed reduction of acetophenone demonstrated a dependence of the initial 


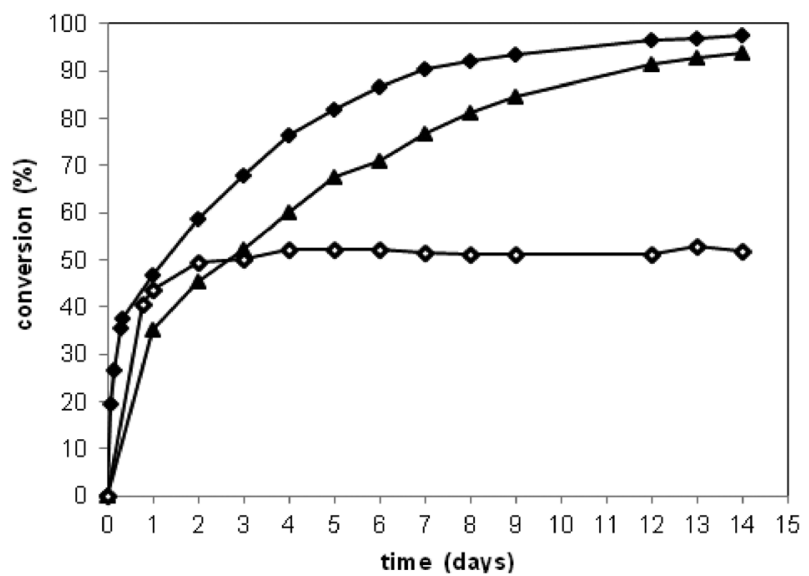

Fig. 2 Conversion of acetophenone by $E$. coli whole cells with overexpressed CPCR in neat substrates. Different volumetric ratios of isopropanol to acetophenone were set $(50: 50 \boldsymbol{\Delta}, 70: 30 \diamond \diamond)$. Closed symbols: daily removed acetone; open symbol: no acetone removal.

reaction rate on the volumetric substrate: co-substrate ratio, indicating the underlying equilibrium conditions (Fig. 2).

Nevertheless, the conversion of acetophenone was always driven to completion, when acetone was intermittently removed from the reaction, and the enantioselectivity was always at maximum (ee $>99 \%){ }^{20,21}$ Obviously, with a highly optimized process development these full conversions could be achieved in much shorter reaction times. Remarkably, after 14 days of operation in the system cells still displayed activity rendering this biocatalyst extremely stable. ${ }^{22}$ This is consistent with the reported stable operation of $E$. coli cells harboring an alcohol dehydrogenase at high concentrations of isopropanol in aqueous media, ${ }^{23}$ and can mainly be attributed to the enzyme preserving microenvironment of the host cell. ${ }^{5}$ Inhibition or inactivation of the catalyst at elevated co-substrate concentrations could not be observed. Consequently, with this system it was possible to produce up to $500 \mathrm{~g} \mathrm{~L}^{-1}$ optically pure $(S)$-phenylethanol. Other substrates like 4-chloroacetophenone or aliphatic 2-butanone were converted in analogous systems as well.

The role of the water on the activity of the biocatalyst was analyzed in depth, since it is widely accepted that water plays a crucial role in biocatalysis in non-conventional media, ${ }^{26}$ in lyophilized whole cells in gas-solid reactor systems, ${ }^{27,28}$ and in whole cell biocatalysis in transesterification and trans-glucosylation reactions. ${ }^{29,30}$ Lyophilized cells were equilibrated to different $a_{\mathrm{w}}$ values ranging from dry $\left(a_{\mathrm{w}}=0\right)$ to wet $\left(a_{\mathrm{w}}=1\right)$ and used in neat substrates (Fig. 3). ${ }^{24,31}$ For low $a_{\mathrm{w}}$ values biocatalytic activity is increasing with water activity, which is in agreement with former findings. ${ }^{27,28}$ The optimum water activity is around an $a_{\mathrm{w}}$ value of 0.84 (Fig. 3). Additionally, non-equilibrated wet cells were active but rapidly deactivated within few hours, whereas lyophilized cells remained stable for several days (data not shown).

As indicated previously, application of the herein described concept may be particularly beneficial for the asymmetric reduction of compounds exhibiting low stabilities in aqueous environments. For instance, propargylic ketone 3-butyn-2-one is unstable in aqueous media, shows thermal decomposition

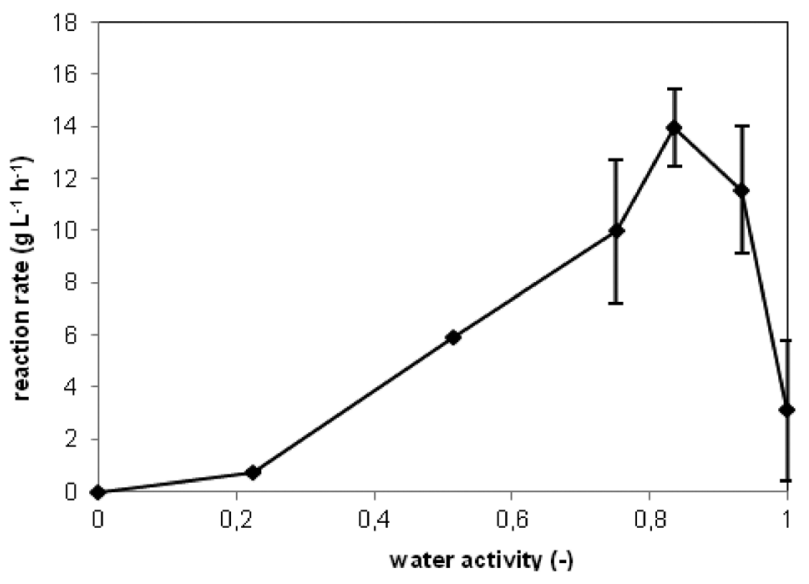

Fig. 3 Initial reaction rate of acetophenone in neat substrates employing E. coli whole cells with overexpressed CPCR. Dried cells and reaction mixtures were previously equilibrated to different water activities. ${ }^{24,25}$

and, moreover, has been reported to deactivate enzymes and cells. ${ }^{32-34}$ Notably, the corresponding (S)-3-butyn-2-ol is an important building block for anti-asthma drugs like 5-lipooxygenase inhibitors, ${ }^{34}$ the potent $\beta_{2}$-adrenergic stimulant broxaterol ${ }^{35}$ or the protease inhibiting hydroxyethylene dipeptide isosteres. ${ }^{36}$ In virtue of this importance, the enzymatic production of $(S)$-3-butyn-2-ol has already been attempted via the asymmetric reduction of 3-butyn-2-one with alcohol dehydrogenases or via the enantioselective hydrolysis of the corresponding esters. To overcome substrate and product instability in aqueous media, either biphasic systems were applied, or the derivatized trimethylsilane-based compound was used as a substrate, albeit at the cost of adding further synthetic steps. ${ }^{33-35,37}$

So far, alcohol dehydrogenases displayed low-to-moderate enantioselectivities in monophasic systems $\left(5-86 \%{ }^{32-35}\right)$, whereas the hydrolase-catalyzed ester hydrolysis led to high ee' $s$, but with limited conversions at $50 \%{ }^{38,39}$ To date, there is only one multi gram-scale preparation of $(S)$-3-butyn-2-ol using an alcohol dehydrogenase from Pseudomonas aeruginosa in a biphasic system with ee $>99 \%$, though at low conversion $(45.5 \%){ }^{32}$

In the herein developed solvent-free whole-cell system high enantioselectivities were achieved (ee $=99.9 \%$; Fig. 4$).{ }^{19}$ Data are at odds with the value reported by Schubert et al. for CPCR for 3-butyn-2-one (ee $=49 \%) .{ }^{34}$ However, in the mentioned work, CPCR was used not in the recombinant form but as a crude extract from the Candida host. Hence, discrepancies might be explained by the acceptance of 3-butyn-2-one by yeast oxidoreductases other than CPCR which in turn may lower the ee, as it has been reported for other yeast systems like baker's yeast. ${ }^{40}$<smiles>[R]C(C)CC(C)O</smiles><smiles>C#C[C@H](O)[CH+]C(C)=O</smiles>

Fig. 4 Biocatalytic reduction of 3-butyn-2-one using lyophilized E. coli cells with overexpressed CPCR in the developed solvent-free system with isopropanol-coupled cofactor regeneration. 
According to the above-described findings that increasing the co-substrate concentration favors the product formation, a volumetric ratio 90:10 (isopropanol to 3-butyn-2-one) at optimum $a_{\mathrm{w}}$ was set up in this case. As proof-of-concept $67.6 \%$ conversion was achieved in $24 \mathrm{~h}$, corresponding to $57.4 \mathrm{~g} \mathrm{~L}^{-1}$ of optically pure (S)-3-butyn-2-ol. This product concentration is already 2 -fold higher than the so far reported maximum value $\left(24.7 \mathrm{~g} \mathrm{~L}^{-1}\right){ }^{32}$ Without acetone removal (hence, under non-optimized conditions), full conversion could be obtained within $120 \mathrm{~h}$, yielding a product concentration of $87 \mathrm{~g} \mathrm{~L}^{-1}$. This example notably points out that enzymatic reductions in neat substrates, while lacking bulk water, is very powerful in accessing optically pure alcohols, even if substrates and/or products are unstable in aqueous solutions. It can be expected that this will open new biocatalytic routes to the production of so far non- or hardly biocatalytically accessible building blocks.

In summary, lyophilized E. coli whole-cells with overexpressed carbonyl reductases perform enantioselective ketone reductions in neat substrates, producing enantiomerically pure alcohols in large amounts. The strategy is characterized by high cost-effectiveness (high added-value, no cofactor addition, simple work-up) and by an environmentally-friendly operation mode (largely diminished waste production, bio-based catalyst). A further notable point is the use of compounds in the reaction system that are unstable in aqueous environments. Together with inherent advantages of biocatalysis (high selectivity, high activity), the approach may represent a competitive alternative to classical chemical production of optically pure alcohols. Extension of the technology to other enzymes and substrates is currently under development and will be reported in due course.

This work was funded by the DFG research training group GK1166 "Biocataysis in non-conventional media" (BioNoCo).

\section{Notes and references}

1 R. Wohlgemuth, Curr. Opin. Microbiol., 2010, 13, 283-292.

2 S. M. De Wildeman, T. Sonke, H. E. Schoemaker and O. May, Acc. Chem. Res., 2007, 40, 1260-1266.

3 A. M. Klibanov, Acc. Chem. Res., 1990, 23, 114-120.

4 P. Adlercreutz, in Organic Synthesis with Enzymes in Non-Aqueous Media, ed. G. Carrea and S. Riva, VCH-Wiley, Weinheim, 2008, pp. 3-24.

5 C. C. de Carvalho, Biotechnol. Adv., 2011, 29, 75-83.

6 K. Goldberg, K. Schroer, S. Lutz and A. Liese, Appl. Microbiol. Biotechnol., 2007, 76, 249-255.

7 H. Gröger, F. Chamouleau, N. Orologas, C. Rollmann, K. Drauz, W. Hummel, A. Weckbecker and O. May, Angew. Chem., Int. Ed., 2006, 45, 5677-5681.

8 W. Stampfer, B. Kosjek, C. Moitzi, W. Kroutil and K. Faber, Angew. Chem., Int. Ed., 2002, 41, 1014-1017.

9 A. Weckbecker, H. Gröger and W. Hummel, Adv. Biochem. Eng./ Biotechnol., 2010, 120, 195-242.

10 Y. Yan Ni, C.-H. Chun-Xiu Li, J. Jie Zhang, N.-D. Shen, U. T. Bornscheuer and J.-H. Xu, Adv. Synth. Catal., 2011, 353, 1213-1217.

11 K. Nakamura, S.-I. Kondo, Y. Kawai and A. Ohno, Tetrahedron Lett., 1991, 32, 7075-7078.
12 P. Nikolova and O. P. Ward, Biocatal. Biotransform., 1994, 9, 329-341.

13 P. Hoyos, G. Sansottera, M. Fernández, F. Molinari, J. V. Sinisterra and A. R. Alcántara, Tetrahedron, 2008, 64, 7929-7936.

14 F. Molinari, R. Gandolfi, R. Villa and E. G. Occhiato, Tetrahedron: Asymmetry, 1999, 10, 3515-3520.

15 G. de Gonzalo, I. Lavandera, K. Faber and W. Kroutil, Org. Lett., 2007, 9, 2163-2166.

16 H. Yamamoto, N. Kawada, A. Matsuyama and Y. Kobayashi, Biosci., Biotechnol., Biochem., 1999, 63, 1051-1055.

17 D. Gamenara and P. Domínguez de María, Biotechnol. Adv., 2009, 27, 278-285.

18 J. Peters, T. Minuth and M. R. Kula, Enzyme Microb. Technol., 1993, 15, 950-958.

19 Conversion and enantiomeric excess were calculated from the peak areas observed in chiral GC analysis. Assignment of peaks was done with commercial authentic standards.

20 Cells were removed by centrifugation. The cleared reaction mixtures were evaporated under reduced pressure to remove acetone and isopropanol. The remaining mixture of acetophenone and phenylethanol was replenished with isopropanol and the old cells were added.

21 K. Goldberg, K. Edegger, W. Kroutil and A. Liese, Biotechnol. Bioeng., 2006, 95, 192-198.

22 Residual activity of cells after operation under solvent-free conditions was assessed in an aqueous system. Cells were washed in buffer $\left(100 \mathrm{mM} \mathrm{K}_{2} \mathrm{HPO}_{4} / \mathrm{KH}_{2} \mathrm{PO}_{4}\right.$, pH 6.5) and used in $5 \mathrm{~mL}$ of the same buffer for bioconversion of acetophenone $(50 \mathrm{mM}$ acetophenone, $5 \% \mathrm{v} / \mathrm{v}$ isopropanol, $10 \mathrm{mM} \mathrm{NADH}, 200 \mathrm{~g} \mathrm{~L}^{-1}$ cells).

23 K. Schroer, U. Mackfeld, I. A. Tan, C. Wandrey, F. Heuser, S. Bringer-Meyer, A. Weckbecker, W. Hummel, T. Daussmann, R. Pfaller, A. Liese and S. Lutz, J. Biotechnol., 2007, 132, 438-444.

24 L. Greenspan, J. Res. Natl. Bur. Stand., Sect. A, 1977, 81, 89-96.

25 Initial reaction rate was calculated from substrate conversion after 4 hours of operation in neat substrates. The data set was collected from two independent experiments.

26 P. J. Halling, Enzyme Microb. Technol., 1984, 6, 513-516.

27 B. Erable, T. Maugard, I. Goubet, S. Lamare and M. D. Legoy, Process Biochem., 2005, 40, 45-51.

28 T. Goubet, T. Maugard, S. Lamare and M. D. Legoy, Enzyme Microb. Technol., 2001, 31, 425-430.

29 D. Huang, S. Han, Z. a. Han and Y. Lin, Biochem. Eng. J., 2011, DOI: $10.1016 /$ j.bej.2010.08.009.

30 M. Y. Rather, S. Mishra and S. Chand, J. Biotechnol., 2010, 150, 490-496.

31 Water activities of dry substrate solution and cells were established by equilibration for least 48 hours with saturated salt solutions.

32 S. M. De Wildeman, WO2008074506, 2008.

33 C. Heiss and R. S. Phillips, J. Chem. Soc., Perkin Trans. 1, 2000, 2821-2825.

34 T. Schubert, W. Hummel, M. R. Kula and M. Müller, Eur. J. Org. Chem., 2001, 4181-4187.

35 M. De Amici, C. De Micheli, G. Carrea and S. Spezia, J. Org. Chem., 1989, 54, 2646-2650.

36 A. K. Ghosh, N. Kumaragurubaran, C. Liu, T. Devasamudram, H Lei, L. M. Swanson, S. V. Ankala, J. Tang, and G. M. Bilcer, EP1871739, 2009.

37 W. Y. Lou, L. Chen, B. B. Zhang, T. J. Smith and M. H. Zong, BMC Biotechnol., 2009, 9, 90.

38 M. Schmidt, D. Hasenpusch, M. Kahler, U. Kirchner, K. Wiggenhorn, W. Langel and U. T. Bornscheuer, ChemBioChem, 2006, 7, 805-809.

39 K. Nakamura, K. Takenaka and A. Ohno, Tetrahedron: Asymmetry, 1998, 9, 4429-4439.

40 S. Rodríguez, M. Kayser and J. D. Stewart, Org. Lett., 1999, 1, $1153-1155$. 